\title{
Experimental Design and Comparative Testing of a Hybrid-Cooled Computer Cluster
}

\author{
Amanda Bonnie \\ Los Alamos National Lab \\ P.O. Box 1663 , MS B272 \\ Los Alamos, New Mexico \\ noranzyk@lanl.gov
}

\begin{abstract}
With water cooling becoming an affordable option both at home and at scale, it is important to consider the possible benefits over air cooling. There are several methods of liquid cooling, notables include: immersion, cold water cooling, and warm water cooling. The total cost of ownership is difficult to determine with these options as each has a different impact on the data center. Considering retrofit, over a new data center, introduces unforeseen variables that make cost analysis a challenge. Besides the added costs of additional infrastructure, and the cost to remove old, the upfront costs could be daunting. Therefore a cost analysis would be a study of its own. This study however hopes to reveal the resulting tradeoffs in temperature, performance, and power usage presented in the case between classical airflow based heat sink mechanisms to water provided directly at the heat sink. Having control over a discrete chiller will provide answers to the CPU temperatures, power usage, and performance at various inlet water temperatures. To water or to air?
\end{abstract}

\section{Categories and Subject Descriptors}

B.0 [Hardware]: General; B.8.0 [Hardware]: Performance and Reliability-General; C.0 [Computer Systems Organization]: General

\section{General Terms}

Experimentation, Measurement, Performance

\section{Keywords}

water cooling, power savings, varied temperatures, performance changes, water vs. air cooling, data center, compute cluster, CPU throttling

\section{INTRODUCTION}

The driving factors for this research are: 1. HPC is growing, and the race to exascale is here; the machine room and/or data center size is expanding; 2 . Cluster density is increasing producing more

This paper is authored by an employee(s) of the United States Government and is in the public domain. Non-exclusive copying or redistribution is allowed, provided that the article citation is given and the authors and agency are clearly identified as its source.

E2SC2015, November 15-20 2015, Austin, TX, USA

ACM 978-1-4503-3994-0/15/11

DOI: http://dx.doi.org/10.1145/2834800.2834803 to cool; 3. The drive to maintain a low PUE, mandated by the DOE; 4. Total cost of ownership concerns, as cooling data centers is expensive; nearly $30 \%$ of a data center electricity bill is spent on cooling [12].

There are many facets of water cooling, with at least two at odds with one another. One is driven by resiliency. Cooler electronic components last longer, so keeping them colder will increase their lifespan [15]. A thought is that cooler components, such as the $\mathrm{CPU}$, can also produce better results through maintaining higher clock speeds longer [14].

The other is driven by cost. Warm water cooling allows for warm (usually around $45^{\circ} \mathrm{C}$ ) to be delivered via direct to chip methods. This means less work needs to be done by chillers and cooling towers to cool the water down. In some cases it can provide what is known as "free cooling" in which no work needs to be done to cool the water going into a system [12]. "Hot-water" cooling also falls in this "free cooling" regime at much higher inlet temperatures [13]. Some means of warm water cooling also use the waste heat from the computer side water loop, also known as the process loop, to effectively return a heat source for heating buildings and or water.

Saving energy is a desirable outcome from water cooling, but running more, and or longer, cycles is desirable as well. One must rationally consider this trade off based on individual driving factors of a clusters purchase. These factors can include, the workload of the cluster, the concerns for down time, the expected lifetime the machine must exist, and the environment of the current data center it will live in.

Power Usage Effectiveness (PUE) is a term coined by The Green Grid [3] used to measure how efficiently a data center uses energy. The value is given by a ratio of the Total Facility Energy to the IT Equipment Energy [3]. PUE is often a value for argument as it is often difficult to accurately determine. Human error such as not remembering to include the computer room lighting, as well as the difficulty to obtain specific power measurements for IT or facility components, are two common issues associated with determining PUE. Nonetheless, the Department of Energy still has a mandate for all federal data centers to be at or below 1.4 [18].

The novelty of this research was perhaps not realized until measured results were achieved. One of the important concepts of applying High Performance Computing (HPC) to physical simulations is the requirement for tightly coupled applications in which the slowest core brings down the performance and increases time to completion. This reduction in variation, known as "jitter", could significantly increase the performance of typical HPC jobs. The larger the scale, the larger the jitter; therefore wasting more machine time, and more power. 


\section{THEORETICAL BACKBONE}

\subsection{Costs: Running the Water Cooling Sys- tem}

There is still an associated cost with running the water cooling system. Even if "free cooling" was obtained through not chilling the water on inlet, there would still be a cost associated with running the pumps to move water through the system. This section is specific to the Tiered Active Multi-dimensional Indexed Record Store (TAMIRS) cluster and data center explained in Section 3

The Cooler Distribution Units (CDU) and vacuum pumps (further discussed in Section 3.2.2 use power to move water through the TAMIRS water system. Considering a total cost evaluation, the power usage of these devices becomes a necessity. Each vacuum pump uses $400 \mathrm{~W}$ and each CDU uses $75 \mathrm{~W}$ when in primary use. Only one CDU and one vacuum will ever be in primary use; however, the standby power for the others is still measurable. Running properly, it can be said that the cooling system consumes $515.5 \mathrm{~W}$.

The conventional way to cool a data center is through Computer Room Air Condition (CRAC) units [14]. These units often are most efficient with warmer return air temperatures [9]. The electricity costs of cooling systems can account for $30 \%$ of the total electricity bill for operating a data center [26]. The CRAC units provide a simple example of cost savings to be had in both power and monetary value if less air cooling was needed.

Through another study by the author, performed in the same data center as TAMIRS, it was determined that each of the 18 CRAC units consumes $19.46 \mathrm{~kW}$ of power, for a total of just over $350 \mathrm{~kW}$. A CRAC unit also contributes an additional heat load to the data center through its motors. This amount is about $300 \mathrm{~kW}$ of heat load to the room for all 18 units [9].

Each unit has the ability to cool between $114-120 \mathrm{~kW}$ of load. With 18 units in the room, the total cooling capacity is $2069 \mathrm{~kW}$. If the room is running at full capacity the cost to run the CRAC units, not factoring water costs, the motors in the CRAC units pull nearly $260 \mathrm{~kW}$. In a day, at $\$ 0.1256 / \mathrm{kW} / \mathrm{h}$ (the national residential average) [20], approximately $\$ 780$ is spent just running the CRAC units per day. In a year that is nearly $\$ 286,100$ spent on electricity.

Unfortunately this does not provide a direct comparison between cost of air cooling and cost of water cooling. This is difficult if not impossible to even theorize on the iso-basis of cooling and or cost to operate. In a data center the costs could easily be portrayed in favor of either air or water depending on what was chosen to represent the situation. For this reason an example of the full costs of a data center were provided in comparison to that of the single cluster.

\subsection{Reducing Power Use: Fans Small but No- table}

Not only are fans a noise hindrance in the data center, they are also an additional source of power use in a chassis. Server nodes often have multiple fans for cooling both the CPU and other onboard components. These fans often ramp up and down with temperature, which allows them to save a little power when not running full speed. However, because some jobs tend to spike in temperature faster than some controllers can adjust, some clusters are set to run full speed all the time.

The Dell R920 nodes in the TAMIRS cluster (further detailed in Section 3 do not require the fans to run at full speed all of the time; however, as needed, the fans do spin up to full. Each has six Nidec UltraFlow 12VDC, 2.31A, fans capable of moving 158 CFM each. At full speed, these fans pull nearly $166 \mathrm{~W}$ per node. That totals to $3 \mathrm{~kW}$ for just 20 nodes. A typical HPC cluster can have thousands of nodes; Trinity, LANL's next super computer will have over 19,000 nodes [21]. Assuming the power of two of these fans in each of the Trinity nodes, it can be approximated that $7 \%$ of power consumption would be due to fans alone.

One hope from this study is to show that with direct water cooling power can be saved by not needing to spin up these fans, and can help reduce the number of fans needed in the system. Essentially if one could reduce the number of fans needed (or the amount a fan needs to run at full speed), a savings for both power and ones ears could be attainable.

\subsection{Electronic Reliability and Longevity}

Temperature affects both the reliability and longevity of electronic devices. As the density of compute increases the chip temperature is also increasing. An increased temperature leads to higher power consumption via leakage current and results in higher power consumption over all [15].

The reliability of a chip is said to reduce exponentially as temperature increases as a function of $e^{\left(-E_{a} / k T\right)}$, where $E_{a}$ is the activation energy for the failure mechanism being accelerated by increased temperature, $k$ is Boltzmann's constant $(8.617 \mathrm{e}-5 \mathrm{ev} / \mathrm{K})$, and $T$ is temperature in degrees Kelvin. At elevated temperatures silicon devices can fail catastrophically [15].

The life of an electronic device is directly related to its operating temperature. It is said that every $10^{\circ} \mathrm{C}$ temperature increase, from the operating conditions, reduces a component's life by $50 \%$ [10]. The Arrhenius Equation is typically used to model the acceleration of temperature dependent physical processes that lead to a function of wearout [25].

The general case is used to determine a components operating life time at room temperature. Therefore the comparative form of the Arrhenius Equation is used to determine an Acceleration Factor for a change in temperature [25]. Equation 1 gives the Acceleration Factor, where $T_{1}$ is the reference temperature (often room temperature $25^{\circ} \mathrm{C}$ ) and $T_{2}$ is the actual use temperature.

$$
\text { Acceleration Factor }=e^{\left[\left(\frac{E_{a}}{k}\right)\left(\frac{1}{T_{1}}-\frac{1}{T_{2}}\right)\right]}
$$

Even if silicon devices do not fail catastrophically at higher temperatures the electrical characteristics are still experiencing frequent intermittent and even permanent changes [15]. Manufactures of computer hardware often specify a maximum operating temperatures; most devices are rated to function properly up to that specified temperature [6]. Exceeding these temperatures in a chassis can easily occur on a loaded down compute node. Although the CPU itself might not be reaching its thermal limit to throttle, the other components in the system could be reaching their manufacturing limits. This can result in memory errors (though easily found with ECC memory), disk read-write errors, and other problems [6].

It seems logical to conclude that cooler components will result in higher reliability, greater longevity, and lower power use over the entire node. It can also be thought that removing heat through water rather than air also means the node temperature itself could be reduced greatly by not pushing hot air removed from the CPUs through the node. This leaves the fans capable of both moving cooler air, as well as more of it, across the entire chassis. This could increase the lifetime of all of the components.

Directly associated with temperature is leakage current. Leakage current is seen as an increase power use and is often caused by hotter component temperatures [15]. Therefore reducing the temperature of a chip will also result in less leakage power and hence better power performance [14]. 


\subsection{Jitter and Noise}

Variations in performance in a cluster can be a major contributor to performance issues for HPC applications. Jitter, also known as noise, can be introduced through various means, including the operating system, CPU, and any component that can introduce a variance [7]. HPC applications in general are more susceptible to noise because they tend to be tightly coupled across the entire job. This means that a single slow core out of an entire cluster can cause the entire cluster to run at the speed of that slow core, thus slowing down the job time and performance of the overall job running across the cluster [17]. Therefore reduction of this variance can result in an improvement in overall performance and power efficiency.

\section{TESTBED OVERVIEW}

TAMIRS is a Los Alamos National Laboratory (LANL) testbed cluster for use in the exploration of next generation tiered storage technologies. This testbed is composed of 22 Dell PowerEdge R920 servers, 2 custom SuperMicro servers, 4 Dell PowerEdge R720 management nodes and an assortment of networking/management/storage equipment. This cluster presents an interesting opportunity to explore the use of direct-to-chip cooling technology while allowing for experimentation without much interruption of other testing.

The rack configuration of the TAMIRS cluster is shown in Figure 1 There are ten R920 nodes in Rack 2 and ten R920 nodes in Rack 4. These two racks contain only the R920 nodes and thus are desirable for direct comparison of air cooled versus water cooled. Rack 2 will remain "stock" and will run with the original heat sinks shipped from Dell. The airflow for the standard rack setup is standard from front to back utilizing $25 \%$ perf tiles. Because this cluster is in production and will need to provide cycles to other users, only four nodes will be retrofit to run the water blocks in the lower section of Rack 4. Nodes 11, 12, 13, and 14 will be water cooled and directly compared to its equal air cooled nodes (same height and location in Rack 1), nodes 1, 2, 3, and 4.

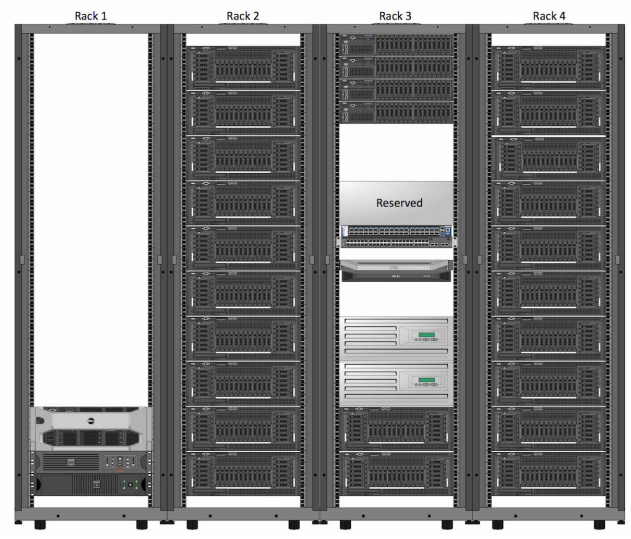

Figure 1: TAMIRS rack layout diagram.

The R920 provides a unique heat sink design from factory (shown later in Section 3.2.4. Tight clearances and tolerance requirements for design of the water block design lead to the need for a custom solution. LANL collaborated with Chilldyne, Inc. [11] to achieve a workable water block design. Section 3.2 covers this design in detail.

\subsection{Full System Specs}

\subsubsection{Software}

The operating system (OS) running on TAMIRS is Tri-Lab Operating System Stack (TOSS) 2.2-3, a common grown OS between the tri-labs (LANL, Lawrence Livermore National Lab (LLNL), and Sandia National Lab (SNL) as a derivative of Red Hat Enterprise Linux (RHEL)). It has similarities between LANL production cluster in the sense that users obtain jobs via the Moab scheduler, and have access to module files and a shared NFS file system for access to users home and project directories. Additional software utilized for monitoring or testing will be discussed in detail in Section 4

\subsubsection{Hardware}

The R920 is unique in that it is a high-density node that is capable of supporting $4 \mathrm{CPU}$ sockets and up to $6 \mathrm{~TB}$ of RAM (in the form of 96 - 64GB DIMMS). The R920 nodes in TAMIRS are half populated with only two of the four sockets populated. The two processors are Intel Xeon E7-4870 v2 at $2.30 \mathrm{GHz}$ with a Turbo clock of $2.90 \mathrm{GHz}$. Each has 15 cores with 30 total threads, for a total of 60 threads per node. The Thermal Design Power (TDP) is $130 \mathrm{~W}$ [4] with a critical threshold temperature of $73^{\circ} \mathrm{C}$. Turbo mode and hyperthreading both remained enabled.

Each node is comprised of eight 16GB (1600MHz RDIMM dell\#3314428 ) sticks of RAM for a total of $128 \mathrm{~GB}$ per node. Each node also has three $1100 \mathrm{~W}$ (dell\#450-AAVK) power supplies in a $2+1$ redundancy configuration. This means that two power supplies provide power to the node normally, while an extra is ready to go online if a power supply failure occurs. These power supply units (PSU) are rated 80 PLUS Titanium on the 80 PLUS Certification. The 80 PLUS Certification requires that PSUs be $80 \%$ or greater energy efficient at $10,20,50$, and $100 \%$ of rated load with a true power factor of 0.9 or greater [23].

There are additional hardware components in each node, but are not relevant to this document as they will not be utilized during testing.

\subsection{Chilldyne Setup}

The Chilldyne system is unique in the field of water-cooling due to the fact that it works under vacuum (negative pressure) rather then positive pumping pressure. This means that the water is pulled through the system, through the manifolds, and water blocks, rather than pushed. Because of this design the entire system is less prone to leaks or spills on equipment. If a line is cut or removed from a manifold or node on purpose or by accident, the vacuum pulls the water back into the system loop and at most a droplet of water results. Traditional systems under pressure will spray out water from a cut or unplugged line and not stop until the pump is stopped or out of water. This makes the Chilldyne a preferred choice in a data center where leaking water could have an effect on several system besides the cluster itself. It also provides assurance that the hardware is safe even when the system is not under visual inspection $24 \times 7$.

The following configuration is Chilldyne's smaller configuration deemed the "demo unit". The full scaled system is capable of cooling up to $200 \mathrm{~kW}$ while consuming only $3 \mathrm{~kW}$ of power. This would effectively be a $1.5 \%$ power consumption for the amount of cooling power produced. The "demo unit" on the other hand uses, as explained prior, $515.5 \mathrm{~W}$ of power. Though the exact cooling capability of the "demo" has not been released, it is more than enough to easily cool the entire TAMIRS cluster at over $6 \mathrm{~kW}$ full load.

\subsubsection{Standalone Chiller}

The standalone chiller unit is a ThermoFlex 7500. Its standard operating temperature range is between $5^{\circ} \mathrm{C}$ and $40^{\circ} \mathrm{C}$. Due to the 
dew point $\left(55^{\circ} \mathrm{F}\right.$ or $\left.\sim 12^{\circ} \mathrm{C}\right)$ and the desire to avoid condensation within the nodes, a safe minimum setting was determined to be $65^{\circ} \mathrm{C}$. This chiller unit could (and ideally would) be replaced with a connection to facility water; a standalone chiller was used for this study to enable the control of water temperature set points outside the available facility water lines. In either case, the standalone chiller or facility water, the system effectively has two loops. An external loop provides the chilling (which can follow less strict water requirements) and an internal loop is feed by the CDUs to the cluster itself. Figure 2 shows both the chiller and CDU configuration.

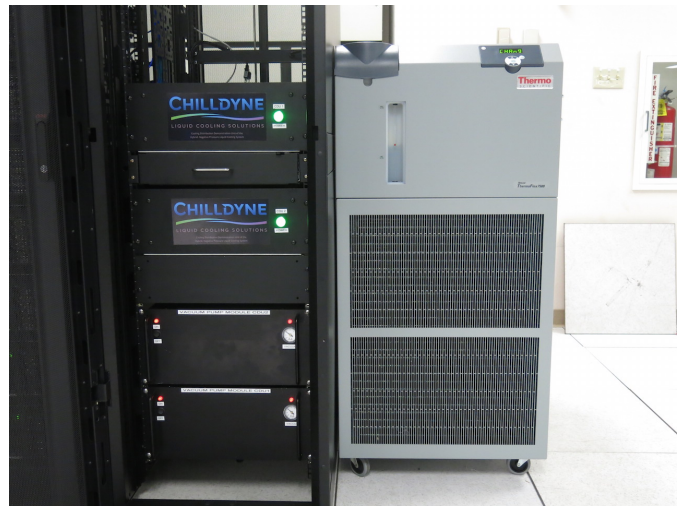

Figure 2: Image of the CDUs with vacuum pumps (left), and standalone chiller (right).

\subsubsection{Cooler Distribution Units \& Vacuum Pumps}

Chilldyne has provided a full rack with two Cooler Distribution Units (CDU), and two vacuum pumps. This is run in a $1+1$ redundancy configuration, with one vacuum pump per CDU, and currently being run with manual fail-over valve to switch to the secondary CDU and pump if a failure were to occur. An automatic failover valve is still under development at Chilldyne.

The pump in the CDU was developed by Chilldyne's sister company, Flometrics. It was originally designed as a rocket fuel pump. It uses two pumping chambers which are alternately filled with fluid and pressurized in sequence to maintain a steady flow of pressured fluid [1]. This is what allows the system as a whole to run at negative pressure.

As previously stated this deployment is a scaled down version of a larger system where the CDU and vacuum pumps are capable of handling a $200 \mathrm{~kW}$ thermal load while dissipating $3 \mathrm{~kW}$ of power themselves. The small units in use for this testing have not been evaluated for an absolute thermal load.

\subsubsection{Rack Manifolds}

There are water lines from the chiller to the CDU, but the CDU still needs a route out to the racks. In each of the three racks (Racks 2,3 , and 4 ) there are two aluminum $\sim 1 / 2$ " square manifolds. Each has a total of 12 stub outs to connect tubing to nodes. One manifold is used for supply, the other for return. The manifold stub outs not in use can be simply capped off with rubber caps to maintain the system vacuum. These can be seen on the manifolds in Figure 3 These manifolds are connected to each other between racks via waterlines beneath the floor.luz

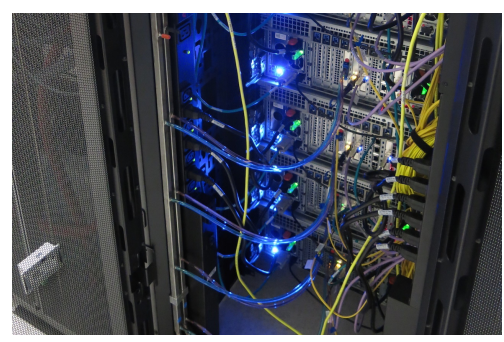

Figure 3: Image of the manifolds mounted in rack. Orange and black rubber caps block off the stub outs not in use.

\subsubsection{Waterblocks}

The Dell R920 posed a challenge for a water block design. The original heat sink for the nodes was a copper block with heat pipes extruding out away from the CPU into a set of copper fins. Although it appears as a traditional heat sink, the space to access the CPU was small and had tight tolerances to allow for additional hardware to fit in the node. Because of this Chilldyne had to custom make the water block from an iterative stage. Figure 4 shows the original heat sink on the right, and plastic prototypes for the new water block design to take its place.

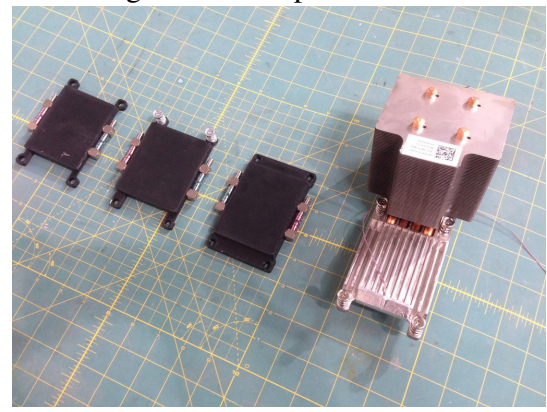

Figure 4: Chilldyne, Inc. designed water blocks (left) to replace the R920 heat sink (right)

After fit was verified the design was sent out to be fabricated from copper blocks. The resulting blocks that are actually installed in the TAMIRS cluster are shown in Figure 5 Because each node has two CPUs populated, the water blocks were run in parallel to ensure that all CPUs would receive the same inlet water temperatures at the blocks themselves. This eliminates any temperature differential across the CPUs that would normally occur in a serial configuration.

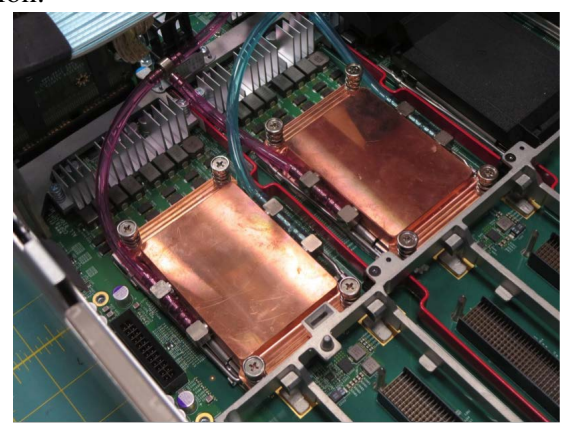

Figure 5: Final water block design for the Dell R920.

\section{TESTING SETUP}

\subsection{Testing Configurations}

The testing programs and configurations are detailed in the fol- 
lowing sections. One integral part of the experimentation was testing the variance in the water supply temperature from the chiller unit. Because the chiller temperature was controllable, both air cooled, and water supply temperatures of $65^{\circ} \mathrm{F}$ and $75^{\circ} \mathrm{F}$ were tested.

\subsection{Stress Testing Nodes}

It was important during testing execution to provide a stress level on the nodes comparable to a representative computational task to provide usable results. It was also important to design controlled and repeatable test(s) to be performed across testing runs. Pavillion is a test harness under development at LANL which allows the same configurations of a test suite to be run again and again with minimal efforts.

\subsubsection{Pavillon}

Pavillon allows for a test suite to be configured to help insure the same performance metrics are measured between different testing configurations as well as different testing runs. Through this testing harness two tests have been built and configured, HPL and Systemburn. The appropriate configuration files for both Pavillon and the individual tests are included in the subsequent sections. Once Pavillion is configured, the different test configuration can be run using the following command:

pav run_test_suite configFile.yaml -m

This command invokes Pavilion (pav) to run the test suite defined by a given configuration file (which is written in yaml). The $-\mathrm{m}$ option toggles the metrics option which allows for collection of data via the Lightweight Distributed Metric Service (LDMS, see Section 4.3.1). This starts the job when the requested nodes are available through the Moab scheduler. STDOUT and STDER from the jobs run is located in a subdirectory specified by date, test name, and the specific Moab job id. The LDMS data is sent to another directory which is also specified by the Moab job id. This provides a logical placement of output data that can later be correlated.

\subsubsection{HPL}

The High Performance LINPACK (HPL) benchmark, also referred to as LINPACK, is common for testing CPU stability as well as cluster performance in the HPC world. The Top 500 uses the LINPACK benchmark as a yardstick of performance to compare some of the top rated Supercomputers [5]. The benchmark reflects a clusters "performance of a dedicated system for solving a dense system of linear equations [5]."

Because HPL is a CPU intensive test, it tends to use more power and get the CPU hotter than many other tests. Therefore it was chosen as a focused test that would show the direct effects of the single change from air cooling to water cooling.

The parallel implementation of HPL can be configured to run on multiple nodes over a variety of cluster structures. The HPL.dat file is configuration file in which parameters can be tweaked for best GFLOP performance values. Tuning this file can take several runs to perfect. The main factors that effect how the file is configured are based on the number of nodes desired to run on, the number of cores per node, and the amount of memory per node. Using a simple HPL.dat file tuner available online from Advanced Clustering Technologies Inc. [2] provided an adequate configuration for testing.

\subsubsection{Systemburn}

Because HPC clusters tend to use more than their CPUs for computation, relying on performance of other hardware components as well, it was important to test "real world" examples to show what effects water cooling may have under typical use. Therefore configurations were built using Systemburn to provide representation of utilization of more than the CPUs on the node(s).

Sytemburn is a software package developed at Oak Ridge National Laboratory (ORNL) designed to allow methodical creation of system loads. Testing "modules" can be configured with different options and combined together to form "LOAD" files for Systemburn to run. Detailed descriptions are available in the documentation for these modules. Only the four used for testing will be explained for sake of brevity: DGEMM, DSTREAM, PV2, and SLEEP. Table 6 provides descriptions for these tests. The "size" parameter will refer to a given parameter in the Systemburn LOAD file.

Table 1: Systemburn module summary, note the "size" parameter described will be used in the LOAD configuration file [16].

DGEMM A double precision matrix multiplication benchmark which will run to consume "size" bytes of memory

DSTREAM Streaming double precision floating point vector operations turn to consume "size" bytes of memory

PV2 A power hungry streaming computational algorithm on one array of 64bit values, which will operate with a memory footprint of "size" bytes. This load was tuned to a quadcore Intel "Nehalem" processor, but may be suitable for loading multiple x $86-64$ cores until the memory system is saturated. It is intended to be run with a footprint large enough to require main memory access.

SLEEP Puts a thread to sleep for N seconds at a time

These load files are dependent on the amount of cores and memory available per node. It was important to think about the configurations for these different tests in detail. For example, two test configurations were created for DGEMM; DGEMM_LARGE* and DGEMM_SMALL for runs with larger amounts of ram and smaller runs respectively. If done with a small enough amount of memory, such to remain in cache (30MB in the case of these nodes) would ultimately result in a warmer running job as more time is spent at the CPU rather than fetching from RAM. The PV2 test takes advantage of this scheme as well. Likewise the larger RAM size assignment meant the job had to hit RAM, which while isn't getting the CPU as hot, is providing example of more real application use. *Note: the larger RAM amounts for DGEMM_LARGE required longer periods of time to complete due to the memory access.

\subsubsection{Running the Tests}

Due to the test run lengths getting in multiple iterations of each test was difficult. To maintain statistical relevance, each of the tests was run a total of 5 times for each of the physical configurations: air cooled, and $65^{\circ} \mathrm{F} / 75^{\circ} \mathrm{F}$ supply water temperatures.

Since the time length of the tests were long, the node was able to reach an equilibrium temperature and run there for most of the duration of the test to provide best representation of temperature, power use, and performance. Each of the tests were also followed by a sleep period in which the node was allowed to reach idle temperature before having to run the next test. The runtime values are shown in Table 2 .

\subsection{Monitoring}


Table 2: Run times for each test suite, recall 24 hours $=1440$ minutes

\begin{tabular}{|c|c|c|}
\hline Test Name & Time to Run [MIN] & $\mathbf{x 5}$ \\
\hline HPL & $\sim 84$ & 420 \\
DGEMM & 105 & 525 \\
DSTREAM & 45 & 225 \\
PV2 & 45 & 225 \\
\hline TOTAL TIME & $\mathbf{2 7 9}$ & $\mathbf{1 3 9 5}$ \\
\hline
\end{tabular}

Measuring temperature would have required an additional script to launch with the jobs collecting data to later be deciphered. Fortunately prior work by the author in development of LDMS@LANL [8] provided an adequate tool for collecting temperature data during a job run in an easily parsable format with date and time. This tool is described in a following subsection.

Measuring power was one of the more difficult metrics to measure. In a data center many clusters may be run from one Power Distribution Unit (PDU). Trying to measure the power of a particular cluster from that PDU can be difficult to factor out. However, rack mounted PDUs are available in metered options. TAMIRS was purchased with per outlet PDUs that allow for individual plug power collection. Running Average Power Limit (RAPL) is an Intel tool used in power capping; it also has the ability to calculate an estimated power use value. These two tools will be used and compared jointly. Details on these monitoring tools is described in the following subsections.

\subsubsection{Temperature: Im_sensors \& LDMS}

The Lightweight Distributed Metric Service (LDMS) tool was developed at Sandia National Lab (SNL) as a data collection and transport system. It provides capacities for lightweight runtime collection of high-fidelity data. Data can be accessed or transported off node via CSV files. This tool is now publicly available on Github under the ovis-hpc project |22|. The LANL version of LDMS (developed to run as a user interface [8]) was used to collect temperature data on the interval of every second during the duration of the tests run by Pavillion.

The package "lm_sensors" is installed on the TAMIRS cluster. This allows for the command line program "sensors" to be called on any node. This outputs the currently reported core temperatures from all 30 physical cores on the node separated by CPU0 and CPU1. The Physical id outputs the maximum of these temperatures at the top of each of the cores. This provides a quick reference for how temperature looks on a node. The LDMS plugin "procsensors" utilizes $1 \mathrm{l} \_$sensors for temperature collection.

\subsubsection{Power: Power Distribution Units}

The cluster configuration allows for power monitoring at the individual plug level with the American Power Conversion Corporation (APC) per outlet metered Power Distribution Units (PDU); therefore the power usage of each node is available. There are a total of six PDUs for the cluster, two per rack. The specific model: AP8641 has a web interface in which all the command line parameters can be set from a web page accessible to the configured IP address. After setting up the interface through serial port connection access to the PDUs was available from the cluster via IP. The PDUs were set to collect power data on a minute interval. This data was sent to the master node of the cluster via an FTP server. This data was collected for each of the PDUs and stored for access by date and time. Parsing of his data for the specific nodes was required.

\subsubsection{Power: Running Average Power Limit}

Intel's Running Average Power Limit (RAPL) driver was designed for power capping [19], however, it also provided a means for power metering. RAPL provides a set of counters for energy and power consumption information. It is a software power model that estimates energy usage by using hardware counters and I/O models [19].

Because power usage is a desirable metric, several scripts have been written for collection of data from the RAPL driver. A program from the University of Maine was written to read the RAPL msr registers containing the power usage and other relevant counter data for Sandy Bridge machines [24]. Due to its access to mrs registers root or sudo access was required for use of this tool.

A script was written to call this program every 30 seconds in an infinite loop. This was launched with the HPL and Systemburn tests to collect power usage data during the lifetime of the job. Any overhead inserted by this collection of data would be invoked equally upon each of the jobs to be compared and thus is said to be negligible.

\subsubsection{CPU Clock Speed}

Throttling by temperature is a reason a job may take longer to complete than expected. Thermal throttling reduces the clock speed of the CPU when a thermal limit is reached. It is a protective method to protect the integrity of the hardware. Using counters from /proc/cpuinfo was not adequate in measuring the changes in CPU speed as the frequency for collection did not capture changes.

\section{RESULTS}

\subsection{Performance}

Performance data between air-cooled and water cooled results are shown in the following subsections. The raw result values were presented for HPL while the Systemburn modules presented results as minimum, mean, and maximum for the run. These resulting values presented are averages over the five runs each.

Table 3: HPL test results, given as average values for, minimum, mean, and maximum.

\begin{tabular}{|c|c|c|c|}
\hline $\begin{array}{c}\text { Cooling } \\
\text { Method }\end{array}$ & $\begin{array}{c}\text { Result } \\
\text { [GFLOPS] }\end{array}$ & STDEV & Improved \\
\hline air & 1247 & 9.34 & -- \\
water $\left(65^{\circ} \mathrm{F}\right)$ & 1257 & 11.04 & $0.80 \%$ \\
water $\left(75^{\circ} \mathrm{F}\right)$ & 1260 & 14.13 & $1.04 \%$ \\
\hline
\end{tabular}

Table 4: Systemburn:DGEMM test results, given as average values for, minimum, mean, and maximum. [MFLOPS]

\begin{tabular}{|c|c|c|c|c|}
\hline $\begin{array}{c}\text { Cooling } \\
\text { Method }\end{array}$ & Min. & Mean & Max. & $\begin{array}{c}\text { Improved } \\
\text { (Mean) }\end{array}$ \\
\hline $\begin{array}{c}\text { air } \\
\text { water }\left(65^{\circ} \mathrm{F}\right)\end{array}$ & 356.19 & 367.44 & 381.63 & -- \\
water $\left(75^{\circ} \mathrm{F}\right)$ & 366.11 & 375.93 & 393.16 & $3.12 \%$ \\
\hline
\end{tabular}


Table 5: Systemburn:DSTREAM test results, given as average values for, minimum, mean, and maximum [MFLOPS].

\begin{tabular}{|c|c|c|c|c|}
\hline $\begin{array}{c}\text { Cooling } \\
\text { Method }\end{array}$ & Min. & Mean & Max. & $\begin{array}{c}\text { Improved } \\
\text { (Mean) }\end{array}$ \\
\hline air & 354.19 & 360.32 & 366.58 & -- \\
water $\left(65^{\circ} \mathrm{F}\right)$ & 348.81 & 360.19 & 367.922 & $-0.04 \%$ \\
water $\left(75^{\circ} \mathrm{F}\right)$ & 353.94 & 361.81 & 367.47 & $0.45 \%$ \\
\hline
\end{tabular}

Table 6: Systemburn:PV2 test results, given as average values for, minimum, mean, and maximum. [MTRIPS/s]

\begin{tabular}{|c|c|c|c|c|}
\hline $\begin{array}{c}\text { Cooling } \\
\text { Method }\end{array}$ & Min. & Mean & Max. & $\begin{array}{c}\text { Improved } \\
\text { (Mean) }\end{array}$ \\
\hline air & 22.49 & 23.47 & 24.13 & -- \\
water $\left(65^{\circ} \mathrm{F}\right)$ & 23.92 & 24.09 & 24.186 & $2.66 \%$ \\
water $\left(75^{\circ} \mathrm{F}\right)$ & 23.90 & 24.08 & 24.192 & $2.60 \%$ \\
\hline
\end{tabular}

\subsection{Temperature}

The average data center room temperature was about $66^{\circ} \mathrm{F}\left(\sim 19^{\circ} \mathrm{C}\right)$ during testing. Variance in this temperature was not controllable due to being a production environment, thus comparative tests between air and water were run during the same time frames.

A key thought to remember while working in both ${ }^{\circ} \mathrm{F}$ and ${ }^{\circ} \mathrm{C}$, is that the changes in temperature do not relate one to one. For example a $10^{\circ}$ change in ${ }^{\circ} \mathrm{F}$ is not the same as a $10^{\circ}$ change in ${ }^{\circ} \mathrm{C}$. In fact the expected difference from a delta in Fahrenheit can be expressed as about $1.8 \mathrm{x}$ the Celsius value. Therefore a difference of $10^{\circ} \mathrm{F}$ correlates to $\sim 5.55^{\circ} \mathrm{C}$ difference. Ideally one would maintain the same units throughout a study, unfortunately the differences in compute hardware and facilities components also disagree in units ( ${ }^{\circ} \mathrm{C}$ and ${ }^{\circ} \mathrm{F}$ respectively) and thus leads to the discussion of both units.

Running with a $65^{\circ} \mathrm{F}\left(\sim 18^{\circ} \mathrm{C}\right)$ water inlet temperature the temperatures during jobs were cooler on average of $\sim 20^{\circ} \mathrm{C}$. At idle the same difference between an air-cooled node and water cooled node was about $\sim 20^{\circ} \mathrm{C}$. In both cases about a $7^{\circ}$ variance between cores was observed between the coldest and hottest core.

The temperature data showed similar differences between HPL and Systemburn runs, therefore, only plots for HPL runs are shown below for sake of repetition. Because the data was collected at a high interval (once per second) a rolling average was used over a 30 second interval to reduce noise in the plots.

\subsection{RAPL}

The RAPL data showed little difference between air cooling and water cooling during the different job runs. This difference could be due to the fact that RAPL uses a calculation to determine expected power usage based on clock speed and the processor details. It is unclear whether temperature is taken into consideration.

RAPL data was collected on 30 second intervals. The data needed to be collected more frequently than 60 second intervals due to the rollover of the counters RAPL uses. Unfortunately, the rollover of counters still incurred during some test runs. Because of this these large negative values were rejected as outliers in the data and removed for plotting and statistical purposes. Again for sake of repetition, only plots for HPL are shown.

\subsection{Power from PDU}

The PDU data was collected every minute, highest frequency for the PDU collection. The data was available as per plug watt values. Each node had three connections to the PDUs and thus had to be summed over three plugs to obtain total power consumption for the node itself. The resulting per node watt value is plotted over time for each of the jobs run. Representative plots for each of tests are shown the the following figures. A blanket statement can be said that a small difference in power was seen between the $65^{\circ} \mathrm{F}$ inlet water and $75^{\circ} \mathrm{F}$ inlet water temperatures.

\subsection{Results Summary}

Measurements during the HPL testing runs showed that the power consumption under water cooling was reduced on average by about $25 \mathrm{~W}$ per node. It was also noted that the maximum power reached during the run was reduced by nearly $30 \mathrm{~W}$ per node.

Power usage during the DGEMM testing showed the mean power consumption reduced by nearly $30 \mathrm{~W}$ by water cooling. The maximum power reached during the run was also reduced by approximately $40 \mathrm{~W}$.

During the DSTREAM runs the average power consumption was reduced by almost $20 \mathrm{~W}$ from air cooling to water cooling. The maximum power reached was reduced by almost $30 \mathrm{~W}$.

The mean power use while running PV2 under water was reduced by just over $20 \mathrm{~W}$. The maximum power reached was reduced by just over $30 \mathrm{~W}$.

Table 7 summarizes the resulting data.

Table 7: PDU Mean Power Data Summary.

\begin{tabular}{|c|c|c|}
\hline Test & $\begin{array}{c}\text { Mean Power } \\
\text { Savings Per } \\
\text { Node }\end{array}$ & $\begin{array}{c}\text { Total Mean } \\
\text { Power Savings }\end{array}$ \\
\hline HPL & $\sim 25[\mathrm{~W}]$ & $\sim 100[\mathrm{~W}]$ \\
DGEMM & $\sim 30[\mathrm{~W}]$ & $\sim 120[\mathrm{~W}]$ \\
DSTREAM & $\sim 20[\mathrm{~W}]$ & $\sim 80[\mathrm{~W}]$ \\
PV2 & $\sim 20[\mathrm{~W}]$ & $\sim 80[\mathrm{~W}]$ \\
\hline
\end{tabular}

\section{DISCUSSION}

The data may not have matched up exactly with expectations presented prior to the results of this study. The following sections detail the observations.

\subsection{Performance}

Performance data between air cooled nodes and water cooled nodes was exactly what was desired. A performance increase would have shown that cooler transistors are able to perform better. However, the particular architecture the study was performed on did not have the ability to turn off power limits to enable these longer bursts of turbo. In this situation the hardware was power limited not thermal limited.

However, in some BIOS on some motherboards, it is possible to not be power limited, and rather be thermal limited for sustaining higher clock speeds. In these situations, water cooling could (and would be expected) to have an effect on performance data.

For the case of this study, having the same exact performance showed that water cooling, even at the warmer temperatures did not negatively effect performance; which was ultimately the desired result.

The surprising result shown in the performance data is actually observed in the minimum performance points shown in the Systemburn data. The minimum performance in the calculation intensive tests, DGEMM and PV2 the minimum performance was increased over air by $4.19 \%$ and $6.27 \%$ respectively. This, while clearly contributing to the increased mean, provides encouraging results on 

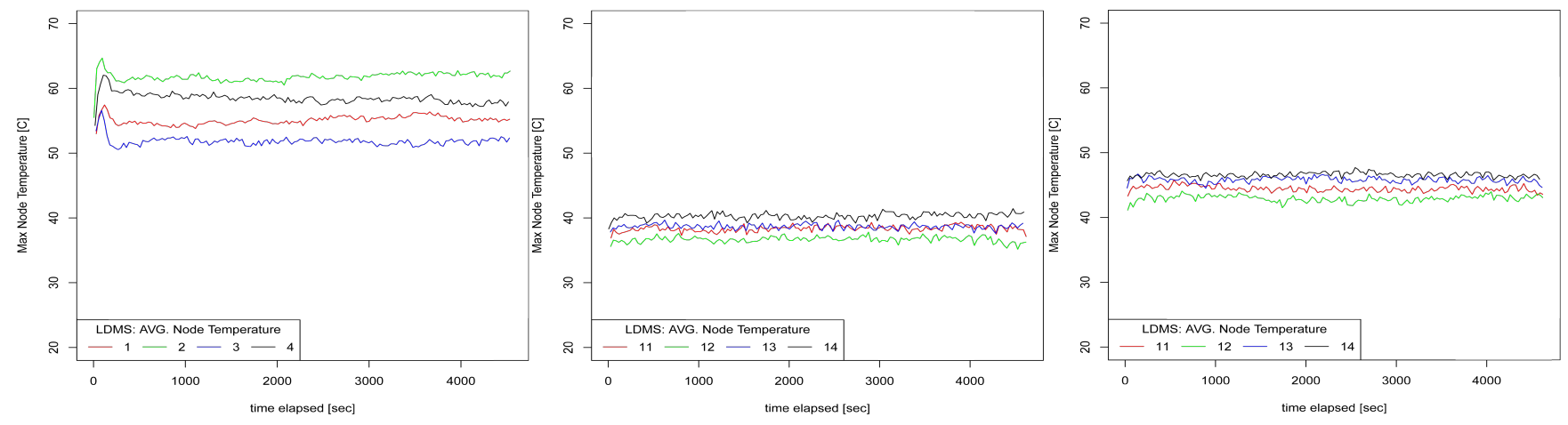

Figure 6: Temperature plots during HPL; air cooled (left), $65^{\circ} \mathrm{F}$ water (center), $75^{\circ} \mathrm{F}$ (right).
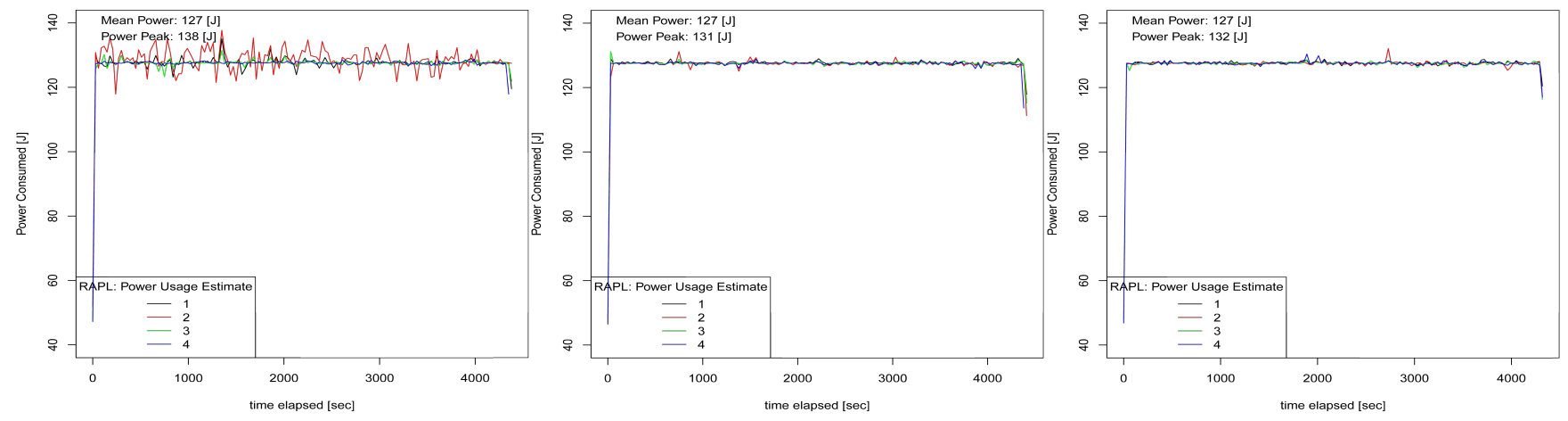

Figure 7: RAPL plots during HPL; air cooled (left), $65^{\circ} \mathrm{F}$ water (center), $75^{\circ} \mathrm{F}$ (right).

overall cluster performance. Considering that a job is always waiting on the slowest core to finish, and cluster wide, the slowest node to finish, this improvement on the slowest one shows better performance overall. On a much larger job, over multiple nodes, this could prove to be a substantial increase in speed, and could result in more than minutes or hours in difference of job time to completion.

\subsection{Temperature}

The difference in temperature between the air cooled nodes and the water cooled nodes was better than expected. Having to have custom blocks designed to fit in such a tight tolerance region, it was unknown exactly what performance would be expected compared to other water block designs from Chilldyne.

Peak temperatures were reduced by about $20^{\circ} \mathrm{C}$ at a $65^{\circ} \mathrm{F}$ inlet temperature and about $15^{\circ} \mathrm{C}$ at at $75^{\circ} \mathrm{F}$ inlet temperature. In this particularly cool data center, the $75^{\circ} \mathrm{F}$ water temperature was actually warmer than room temperature (approx. $66^{\circ} \mathrm{F}$ ); however, even the idle dissipation of the nodes was enough to maintain this water temperature.

Relating this back to the prior section, this further shows that on the right setup, thermal limitations would likely not be the cause for CPU throttling, and rather could remain in turbo mode longer.

Because water is removing most of the thermal load from the node, warmer room temperatures could be obtained without causing temperature issues that may be observed in the air cooled nodes. This warming of the room would reduce facilities costs and increase CRAC efficiency.

\subsection{RAPL}

Not knowing exactly the method used beneath the covers of RAPL, it is thought the RAPL data provided somewhat inconclusive results. The estimated power use between air cooled and the water cooled nodes varied little, though the estimated power usage did seem smoother for the water cooled nodes. Based upon these results it is thought that RAPL may factor in the temperature of the CPU for its calculation. These estimations may or may not be accurate as described in the next section.

\subsection{PDU}

The actual power data collected by the in rack PDUs provided proof of reduced power use between air cooled and water cooled nodes. It is hard to argue whether the power reduction was caused by a reduction in fan speed or perhaps from the CPUs running cooler themselves. If fan voltages could be measured, this could probably be reduced to a root cause for reduction. Nonetheless a power reduction was observed.

Although the $166 \mathrm{~W}$ power reduction from maximum fan speed was not observed a substantial $30 \mathrm{~W}$ per node was observed. It was expected that the fans would still be running, so a $166 \mathrm{~W}$ decrease was an unreasonable to be expected. $30 \mathrm{~W}$ does not seem like a lot of savings, but taken to scale does add up. Over the four nodes in this testing scenario it was a total of about $120 \mathrm{~W}$ saved. Over a 24 hour period this is a savings of $2.88 \mathrm{kWh} /$ day. Though this only adds up to a savings of $\$ 131.61$ in a year on power alone, this does not factor in the effects taken at scale.

Scaled up to the full cluster of a small 20 nodes saves about $600 \mathrm{~W}$, the equivalent of ten $60 \mathrm{~W}$ lightbulbs left on year round. This savings results in $14.4 \mathrm{kWh} /$ day adding up to $\$ 658.05$ in the course of a year. It is easy to see how this can continue to increase in cost savings with scale. 
Though the cost of the Chilldyne system can not be released, the cost for it is not recouped by these power savings alone. Additional savings however could be acquired if this was further taken to the scale of the data center. Returning to the initial thoughts for a 70:30 divided data center significant cost savings could be obtained in reduced facilities costs.

This PDU data, combined with the RAPL results, suggests that the power savings incurred was not due to the CPUs reduction in energy consumption but rather other components of the node. It is thought that perhaps the fans were not spinning up as much during the job. This could be validated through measurement of fan voltages during testing scenarios.

\section{CONCLUSIONS}

Through the culmination of over a year and a half of work; LDMS tool development TAMIRS deployment; configuration, and on going support; Chilldyne contract management and installation; integration of a test suite and monitoring; the results of this study were accomplished.

\subsection{Temperature}

It was determined, at the temperatures tested, that no performance gain was obtained from water cooling alone. However, water cooling did provide cooler CPU temperatures which could allow for higher clock speeds to be obtained. The idle and load temperatures were significantly lower than the air cooled nodes. This provides increased resiliency and lifetime of the components as even running $10^{\circ} \mathrm{C}$ cooler increases the life time of electronic components by double. With HPC clusters often having a home in a data center for at least 5 years, it is important to have resilient components to keep them running while minimizing down time. The easiest way to reduce down time, is to not have the hardware fail in the first place; thus having longer lasting components by providing them with a cooler working environment seems like a start. However, the testing performed did not confirm nor deny extra longevity.

Considering the inlet water temperatures, the warmer water temperature of $75^{\circ} \mathrm{F}$, the CPUs were still able to run $10^{\circ} \mathrm{C}$ cooler than when cooled with $55^{\circ} \mathrm{F}$ air temperatures. The real savings here, which could not be observed at this small scale, would have been the cost savings of the running chiller units, the CRAC units, as well as the cooling towers. It is important to consider the scalable impacts that this smaller study has shown plausible. Reducing the need for cool air cooling in a data center significantly reduces the costs associate with creating the cool air. Large chiller units, and cooling towers are used for producing the $55^{\circ} \mathrm{F}$ air to the data centers. It is possible that chiller units would not even have a part in the process if a data center were to be comprised of water cooled clusters. There may be a need for some comfort cooling; but this would be a minimal cost compared to the costs of maintaining the cold room.

\subsection{Power}

The RAPL data suggested that little to no power efficiency was gained at the chip level. The concepts of leakage current at the temperatures tested did not show in the RAPL results. It is unclear whether or not the RAPL calculations factor in the chip temperature or not; however while under water, the RAPL data did show smoother curves. Assuming the RAPL data was accurate, the power savings at the PDU can then likely be explained by reduced fan speeds in the water cooled nodes. At nearly $30 \mathrm{~W}$ per node in power reduction the reduction of power use over a scaled cluster would be significant. Even at the full capacity of the TAMIRS cluster, this would be over a $600 \mathrm{~W}$ savings. Scaled to a cluster with thousands of nodes, the savings would be even more significant.

\subsection{More}

The warmer water temperatures can be seen as a gateway to "free cooling" with a great savings on the facilities side.In efforts to maintain a specific PUE value while driving towards exascale and its demanding power requirements on the compute and facilities side, the reduction in facility cost via water cooling could provide more power for where it counts; the compute side.

In a data center limited by CRAC unit capacity, systems such as this can be used to expand data center compute capacity far beyond the limitations of the available air cooling. In a full scale system each CDU can handle approximately $200 \mathrm{~kW}$ of thermal load while only using $3 \mathrm{~kW}$ of power. With an external water to air heat exchanger the entire compute thermal load could removed from the CRAC units and other resources (additional storage, networking, etc) could be deployed to utilize the excess thermal capacity. Alternatively, the thermal load savings could be utilized in a way that fewer CRAC units are required which directly lowers the cost of maintaining the data center and its systems.

\section{FUTURE WORK}

Due to time constraints and the desire to graduate, this experimentation and study was limited to the above documentation. However, there are several tests and areas to still be explored. This work will continue despite the completion of this document. A few of the planned testing areas the author wishes to explore are described in the following subsections.

\subsection{Warmer Water Cooling}

Warm water cooling is essentially free cooling. If the water does not need to be chilled before entering the system a lot of money can be saved. Being able to warm up the water and maintain a warmer than room temperature water temperature requires a little more work.

When more water blocks are installed on the system, a "dummy" load can be run on the non-testing nodes. This will provide additional heat in the water loop to help maintain the water temperature between tests as well as during tests that may not produce enough heat to maintain a water temperature.

The plan is to measure warmer water temperatures to see just how warm the water can be before causing issues with throttling at the CPU level. Based on the data presented, it is thought that there is a $20^{\circ} \mathrm{C}$ temperature between the throttling point and where the nodes are running at a $65^{\circ} \mathrm{F}$ water temperature. This relates to a $36^{\circ} \mathrm{F}$ delta in water temperature resulting in $101^{\circ} \mathrm{F}$ water temperature. This is on the upper end of what is supported by the Chiller unit (limited to $104^{\circ} \mathrm{F}$ ) but is still an interesting goal.

\subsection{Tightly Coupled Applications}

Many of the synthetic benchmarks presented here are designed to maximize power usage as well as maximize throughput. Because of this, much of the workload is dominated by compute power and not by synchronized computation. Applications that tend to run on clusters of this design are inherently more coupled and require various synchronization points during the run. These synchronization points depend on the performance of the slowest node, and as such, if the environment for these systems was not as ideal as it was, thermal throttling of an air cooled node could present itself in the form of much reduced system performance for these tightly coupled workloads. 


\subsection{Looking at Scale}

Unfortunately due to time constraints and other considerations the entire cluster was not able to be completely water cooled for this particular research. However, there are plans to install water blocks on the other 20 compute nodes. Additional testing will be performed to see how well this four node test scales out to the entire cluster.

\section{ACKNOWLEDGMENTS}

This work was performed using facilities and resources at Los Alamos National Laboratory and was funded by the United States Department of Defense. This release is under LA-UR-15-26183.

\section{REFERENCES}

[1] Pistonless pump technology. Available: http://www.rocketfuelpump.com.

[2] How do i tune my hpl.dat file? Available: http://www.advancedclustering.com/act-kb/tune-hple-datfile/, September 2014.

[3] The green grid. Available: http://www.thegreengrid.org, Accessed: June 2015

[4] Intel xecon processor e7-4870v2 specifications. Available: http://ark.intel.com/products/75250/Intel-Xeon-ProcessorE7-4870-v2-30M-Cache-2_30-Ghz, Accessed: June 2015.

[5] The linpack benchmark. Available: http://www.top500.org/project/linpack/, June 2015.

[6] Operating temperature vs. system reliability. Availabe: www.firepower-technology.com/support-resources/systemintegrator-resources, Accessed: June 2015.

[7] Beckman, P., Iskra, K., OShit, K., And Coghlan, S. The influence of operating systems on the perforamce of collective operations at extreme scale. Argonne National Laborator W-31-109-ENG-38.

[8] BonNIE, A. Ldms@lanl. Unpublished. Internal source at LANL, May 2015.

[9] Bonnie, A., AND Hefele, D. Ta03-1498-341:optimizing cooling. LANL LA-UR-13-26011, July 2013.

[10] Chen, S., Hu, Y., AND Peng, L. Optimization of electricity and server maintenance costs in hybrid cooling data centers. IEEE 978-0-7695-5028-2 (2013), 526-533.

[11] ChILLDYNE. Liquid cooling technology [online]. Available: http://www.chilldyne.com/, 2015.

[12] Coles, H., Ellsworth, M., and Martinez, D. "hot" for warm water cooling. ACM 978-1-4503-0771-0 (November 2011).

[13] Conficoni, C., Bartolini, A., Tilli, A., Tecchiolli, G., AND BENINI, L. Energy-aware cooling for hot-water cooled supercomputers. EDAA 978-3-9815370-4-8 (2015), 1353-1358.

[14] Ellsworth, M., CAmpbell, L., Simons, R., Iyengar, M., SCHMIDT, R., AND CHU, R. The evoloution of water cooling for ibm large server systems: Back to the future. IEEE 978-1-4244-1701-8 (August 2008), 266-274.

[15] Han, Y., Koren, I., AND Andras MoItZ, C. Temperature aware floorplanning.

[16] Kuehn, J., Poole, S., Hodson, S., Lothian, J., BAKER, M., Dobson, J., ReISTER, D., LEWKOW, N.,
Glandon, R., And Peek, J. Systemburn. Available: https://github.com/jlothian/systemburn, April 2015.

[17] Morari, A., Gioiosa, R., WisnieWski, R., Rosenburg, B., Inglett, T., AND VAlero, M. Evaluating the impact of tlb misses on future hpc systems. IEEE 1530-2075, 12 (2012), 1010-1021.

[18] OF ENERGY, U. D. 1.4 pue - the new mandate for federal data center energy efficiency. Available: http://www.efcog.org/wg/ei/events/Aug_2223_2012_meeting/2012\%20ESSG\%20Training\%20\%Data\%20Center\%20Profiler.pdf, Accessed: June 2015.

[19] PANDRUVADA, S. Running average power limit - rapl. Available: https://01.org/blogs/tlcounts/2014/runningaverage-power-limit-rapl, June 2015.

[20] Power, P. Available: https://www.pacificpower.net/about/rr/rpc.html, August 2014.

[21] Presentation, L. Trinity advanced technology system overview. Available: http://www.lanl.ogv/projects/trinity/_assets/docs/trinityoverview-for-web.pdf:wq, Accessed: June 2015.

[22] Primary: Brandt, J., And Gentile, A. ldms. Available: https://github.com/ovis-hpc/ovis/tree/master/ldms.

[23] Solutions, P. L. 80 plus certified power supplies and manufactures. Available: http://www.plugloadsolutions.com/80PlusPowerSupplies.aspx, May 2015.

[24] WAVER, V., AND DOLBEAU, R. rapl-read.c source code. Available: http://web.eece.maine.edu//vweaver/projects/rapll/raplread.c, November 2013.

[25] Wetterling, S., ANd Barrett, P. Modeling temperature driven wearout rates for electronic components. Available: servernger.com/Resources/Modeling _Temperature_Driven_Wearout_Rates_for_Electronic_Components_b.pdf, Accessed: June 2015

[26] Zhang, Y., WANG, Y., AND XiaORUI, W. Testore: Exploiting thermal and energy storage to cut the electricity bill for datacenter coolin. IFIP 978-901882-48-7 (2012), 19-27. 